PUBLICATIONS DE L'INSTITUT MATHÉMATIQUE

Nouvelle série, tome 74(88) (2003), 71-83

\title{
ON SOME AKIVIS-GOLDBERG TYPE METRICS
}

\section{Ryszard Deszcz}

\author{
Dedicated to Professor Dr. Mileva Prvanović
}

\begin{abstract}
We investigate Akivis-Goldberg type metrics satisfying some additional assumptions.
\end{abstract}

\section{Introduction}

Let $M$ be a manifold of dimension $n=p q$, and let $S C(p, q)$ be a differentiable field of Segre cones $S C_{x}(p, q) \subset T_{x} M, x \in M$. The pair $(M, S C(p, q))$ is called an almost Grassmann structure and is denoted by $A G(p-1, p+q-1)$. The manifold $M$ endowed with such structure is said to be an almost Grassmann manifold (e.g., see [1, Definition 1.1]). Some additional conditions lead to so-called semiintegrable almost Grassmann structures [1, Definition 1.2]. The latter were studied in [1] and examples of such structures, mainly 4-dimensional, are presented there. Certain semi-Riemannian metrics are related to these structures (see Examples 3.5-3.16 of [1]). These metrics are called Akivis-Goldberg, in short $A G$-metrics [20]. Manifolds admitting $A G$-metrics will be called $A G$-manifolds. Curvature properties and, in particular, curvature properties of pseudosymmetry type of $A G$-manifolds were obtained in $[\mathbf{2 0}]$. For instance, on such manifolds we have [20]

$$
\begin{gathered}
\operatorname{rank} S \leqslant 2, \\
\text { (i) } \quad S^{2}=0, \quad \text { (ii) } \quad \kappa=0, \quad \text { (iii) } \quad S \cdot C=0 .
\end{gathered}
$$

For precise definitions of the symbols used, we refer to Section $\mathbf{2}$ of this paper. We note that (1.2)(iii), by making use of (1.2)(i), (1.2)(ii) and the identity

$$
S \cdot C=S \cdot R+\frac{4}{n-2} \bar{S}+\frac{2}{n-2} g \wedge S^{2}-\frac{2 \kappa}{(n-2)(n-1)} g \wedge S,
$$

2000 Mathematics Subject Classification: 53B20, 53B25, 53C25, 83C35, 53A40, 53A60.

Key words and phrases: warped product, pseudosymmetry type manifold, almost Grassmann structure, Akivis-Goldberg metric, Akivis-Goldberg type metric, Akivis-Goldberg type hypersurface, Roter type equation, P. J. Ryan problem.

The author is supported by the grant 2 P03A 00617 from the Polish State Committee of Scientific Research (KBN). 
turns into $S \cdot R=-\frac{4}{n-2} \bar{S}$. Moreover, on every $A G$-manifold $(M, g)$ the following condition of pseudosymmetry type is satisfied $[\mathbf{2 0}]$

$$
R \cdot R-Q(S, R)=L_{C} Q(g, C),
$$

where $L_{C}$ is some function on $U_{C}=\{x \in M \mid C \neq 0$ at $x\}$. With respect to the above presentation of curvature properties of $A G$-manifolds we can define the following extension of this class of manifolds.

Let $(M, g), n \geqslant 4$, be a semi-Riemannian manifold such that $U_{C} \cap U_{S} \subset M$ is a nonempty set, where $U_{S}=\left\{x \in M \mid S-\frac{\kappa}{n} g \neq 0\right.$ at $\left.x\right\}$. The metric $g$ will be called an Akivis-Goldberg type metric, in short an $A G$ type metric if on $U_{C} \cap U_{S}$ the following three conditions are fulfilled: (1.4),

$$
\begin{gathered}
S \cdot R=L_{1} \bar{S}+L_{2} g \wedge S+L_{3} G, \\
S^{2}=L_{4} S+L_{5} g,
\end{gathered}
$$

where $L_{1}, \ldots, L_{5}$ are some functions on $U_{C} \cap U_{S}$. A manifold admitting an $A G$ type metric will be called an Akivis-Goldberg type manifold, in short an $A G$ type manifold. Evidently, every $A G$ manifold is an $A G$ type manifold. The converse statement is not true. In Section 3 we present examples of $A G$ type manifolds. In particular, we state that every semi-Riemannian manifold satisfying the Roter type equation $[\mathbf{9}]$ is an $A G$ type manifold. Some $A G$ type manifolds satisfy also (1.1). In Section 2 we prove (see Corollary 2.1) that if an $A G$ type manifold $(M, g)$ satisfies on $U_{C} \cap U_{S} \subset M$ the condition

$$
\operatorname{rank} S=2
$$

then (1.6) reduces on $U_{C} \cap U_{S}$ to

$$
S^{2}=\frac{\kappa}{2} S
$$

In Remark 3.1 (v) and (vi) we present examples of $A G$ type manifolds satisfying (1.7). These manifolds can be locally realized as hypersurfaces of semi-Euclidean spaces. In the last section we consider hypersurfaces $M$ in semi-Riemannian spaces of constant curvature $N_{s}^{n+1}(c)$ with signature $(s, n+1-s), n \geqslant 4$, or in particular, in semi-Euclidean spaces $\mathbb{E}_{s}^{n+1}$, with nonempty set $U_{C} \cap U_{S} \subset M$, satisfying on this set (1.4), (1.5) and (1.6). It means that the metric $g$ induced on $M$ from the metric of the ambient space is an $A G$ type metric. Hypersurfaces $M$, with nonempty set $U_{C} \cap U_{S} \subset M$, satisfying on this set (1.4), (1.5) and (1.6) will be called Akivis-Goldberg type hypersurfaces, in short $A G$ type hypersurfaces.

Let $M$ be a hypersurface in $N_{s}^{n+1}(c), n \geqslant 4$. We denote by $U_{H}$ the set of all points of $M$ at which the tensor $H^{2}$ is not a linear combination of $H$ and $g$. Using (2.18) and Theorem 4.1 of [19] we can deduce that $U_{H} \subset U_{C} \cap U_{S} \subset M$. AG type hypersurfaces in $N_{s}^{n+1}(c), n \geqslant 4$, are also investigated in [22] and [23]. Among others things in $[\mathbf{2 2}]$ it was shown that (1.4), (1.5) and (1.6) hold on $U_{C} \cap U_{S}-U_{H}$. Therefore we restrict our considerations on $A G$ type hypersurfaces $M$ in $N_{s}^{n+1}(c)$ to the set $U_{H} \subset M$. We mention that an extension of the class of $A G$ type manifolds was introduced in $[\mathbf{2 2}]$ (see also $[\mathbf{2 3}]$ ). 
Our main result states (see Theorem 4.1) that if $M$ is an $A G$ type hypersurface in $\mathbb{E}_{s}^{n+1}, n \geqslant 5$, the set $U_{H} \subset M$ is nonempty, and (1.7) holds on $U_{H}$, then the conditions $R \cdot R=0$ and $R \cdot S=0$ are equivalent at all points of $U_{H}$ at which $\kappa \neq 0$. An example of a semisymmetric $A G$ type hypersurface, with $\kappa \neq 0$, is given in Section 3 (see Remark 3.1(v)). That hypersurface satisfies

$$
R=\frac{2}{\kappa} \bar{S}
$$

\section{Preliminaries}

Throughout this paper all manifolds are assumed to be connected paracompact manifolds of class $C^{\infty}$. Let $(M, g)$ be an $n$-dimensional, $n \geqslant 3$, semi-Riemannian manifold. We denote by $\nabla, R, C, S$ and $\kappa$ the Levi-Civita connection, the RiemannChristoffel curvature tensor, the Weyl conformal curvature tensor, the Ricci tensor and the scalar curvature of $(M, g)$, respectively. The Ricci operator $\mathcal{S}$ is defined by $g(\mathcal{S} X, Y)=S(X, Y)$, where $X, Y \in \Xi(M), \Xi(M)$ being the Lie algebra of vector fields on $M$. We define the endomorphisms $X \wedge_{A} Y, \mathcal{R}(X, Y)$ and $\mathcal{C}(X, Y)$ of $\Xi(M)$ by

$$
\begin{aligned}
\left(X \wedge_{A} Y\right) Z & =A(Y, Z) X-A(X, Z), Y \\
\mathcal{R}(X, Y) Z & =\left[\nabla_{X}, \nabla_{Y}\right] Z-\nabla_{[X, Y]} Z, \\
\mathcal{C}(X, Y)=\mathcal{R}(X, Y) & -\frac{1}{n-2}\left(X \wedge_{g} \mathcal{S} Y+\mathcal{S} X \wedge_{g} Y-\frac{\kappa}{n-1} X \wedge_{g} Y\right),
\end{aligned}
$$

respectively, where $X, Y, Z \in \Xi(M)$ and $A$ is a symmetric (0,2)-tensor. Now the Riemann-Christoffel curvature tensor $R$, the Weyl conformal curvature tensor $C$ and the $(0,4)$-tensor $G$ of $(M, g)$ are defined by

$$
\begin{aligned}
& R\left(X_{1}, X_{2}, X_{3}, X_{4}\right)=g\left(\mathcal{R}\left(X_{1}, X_{2}\right) X_{3}, X_{4}\right), \\
& C\left(X_{1}, X_{2}, X_{3}, X_{4}\right)=g\left(\mathcal{C}\left(X_{1}, X_{2}\right) X_{3}, X_{4}\right), \\
& G\left(X_{1}, X_{2}, X_{3}, X_{4}\right)=g\left(\left(X_{1} \wedge_{g} X_{2}\right) X_{3}, X_{4}\right),
\end{aligned}
$$

respectively, where $X, Y, Z, X_{1}, X_{2}, \ldots \in \Xi(M)$. Let $\mathcal{B}(X, Y)$ be a skew-symmetric endomorphism of $\Xi(M)$ and let $B$ be a $(0,4)$-tensor associated with $\mathcal{B}(X, Y)$ by

$$
B\left(X_{1}, X_{2}, X_{3}, X_{4}\right)=g\left(\mathcal{B}\left(X_{1}, X_{2}\right) X_{3}, X_{4}\right) .
$$

The tensor $B$ is said to be a generalized curvature tensor if

$$
\begin{gathered}
B\left(X_{1}, X_{2}, X_{3}, X_{4}\right)+B\left(X_{2}, X_{3}, X_{1}, X_{4}\right)+B\left(X_{3}, X_{1}, X_{2}, X_{4}\right)=0, \\
B\left(X_{1}, X_{2}, X_{3}, X_{4}\right)=B\left(X_{3}, X_{4}, X_{1}, X_{2}\right) .
\end{gathered}
$$

For a generalized curvature tensor $B$ we denote by $\operatorname{Ric}(B), \operatorname{Weyl}(B)$ and $\kappa(B)$ the Ricci tensor, the Weyl tensor and the scalar curvature of $B$, respectively. The subsets $U_{B}, U_{\mathrm{Ric}(B)}$ and $U_{\mathrm{Weyl}(B)}$ are defined in the same way as the subsets $U_{R}, U_{S}$ and $U_{C}$, respectively. Clearly, the tensors $R, C$ and $G$ are generalized curvature tensors. For symmetric $(0,2)$-tensors $E$ and $F$ we denote by $E \wedge F$ their KulkarniNomizu product. The tensor $E \wedge F$ is also a generalized curvature tensor. For a 
symmetric $(0,2)$-tensor $E$ we define the $(0,4)$-tensor $\bar{E}$ by $\bar{E}=\frac{1}{2} E \wedge E$. In particular, we have $\bar{g}=G=\frac{1}{2} g \wedge g$. Let $\mathcal{B}(X, Y)$ be a skew-symmetric endomorphism of $\Xi(M)$ and let $B$ be the tensor defined by (2.1). We extend the endomorphism $\mathcal{B}(X, Y)$ to derivation $\mathcal{B}(X, Y)$. of the algebra of tensor fields on $M$, assuming that it commutes with contractions and $\mathcal{B}(X, Y) \cdot f=0$ for any smooth function on $M$. Now for a $(0, k)$-tensor field $T, k \geqslant 1$, and a symmetric $(0,2)$-tensor $A$ we can define the $(0, k+2)$-tensors $B \cdot T$ and $Q(A, T)$ and the $(0, k)$-tensor $A \cdot T$. For the definition of these tensors we refer, for instance, to [2] or [13]. Setting $T=R$, $T=C$ or $T=S$ and $A=g$ or $A=S$ we obtain the tensors: $S \cdot R, S \cdot C, R \cdot R$, $R \cdot C, C \cdot R, C \cdot C, R \cdot S, C \cdot S, Q(g, R), Q(g, C), Q(g, S), Q(S, R)$, and $Q(S, C)$. The tensors $C \cdot R, C \cdot C$ and $C \cdot S$ are defined in the same manner as the tensors $R \cdot R$ and $R \cdot S$, respectively.

A semi-Riemannian manifold $(M, g), n \geqslant 3$, is called a quasi-Einstein manifold if its Ricci tensor $S$ has the form

$$
S=\alpha g+\epsilon w \otimes w, \quad \epsilon= \pm 1,
$$

for some function $\alpha$ and 1-form $w$ on $M$. We refer to [2] for a review of results on quasi-Einstein manifolds. $A G$ type quasi-Einstein hypersurfaces in semiRiemannian spaces of constant curvature are investigated in [23].

A semi-Riemannian manifold $(M, g), n \geqslant 3$, is said to be pseudosymmetric if at every point of $M$ the tensors $R \cdot R$ and $Q(g, R)$ are linearly dependent. This is equivalent to

$$
R \cdot R=L_{R} Q(g, R)
$$

on $U_{R}=\left\{x \in M \mid R-\frac{\kappa}{(n-1) n} G \neq 0\right.$ at $\left.x\right\}$, where $L_{R}$ is some function on $U_{R}$. We note that $U_{C} \subset U_{R}$ and $U_{S} \subset M$. The class of pseudosymmetric manifolds is an extension of the class of semisymmetric manifolds $(R \cdot R=0)$. A semi-Riemannian manifold $(M, g), n \geqslant 3$, is said to be Ricci-pseudosymmetric if at every point of $M$ the tensors $R \cdot S$ and $Q(g, S)$ are linearly dependent. This is equivalent to

$$
R \cdot S=L_{S} Q(g, S)
$$

on $U_{S}$, where $L_{S}$ is some function on $U_{S}$. We say that (2.3) and (2.4) are certain conditions of pseudosymmetry type $[\mathbf{2}],[\mathbf{1 2}]$. The class of Ricci-pseudosymmetric manifolds is an extension of the class of Ricci-semisymmetric manifolds $(R \cdot S=0)$ as well as of the class of pseudosymmetric manifolds. Some geometrical considerations show that (2.3), resp., (2.4), is a more natural curvature condition than the condition $R \cdot R=0$, resp. $R \cdot S=0$. For a presentation of facts related to these statements and, in general, on pseudosymmetry type conditions we refer to $[\mathbf{2}]$ and $[12]$.

Lemma 2.1. Let $(M, g), n \geqslant 3$, be a semi-Riemannian manifold and let $A$ be a nonzero symmetric $(0,2)$-tensor at $x \in M$.

(i) If

$$
\operatorname{rank} A=2
$$


at $x$, then at $x$ we have

$$
A^{3}=\operatorname{tr}(A) A^{2}+\frac{\operatorname{tr}\left(A^{2}\right)-(\operatorname{tr}(A))^{2}}{2} A .
$$

Moreover, if

$$
A^{2}=\alpha A+\beta g, \quad \alpha, \beta \in \mathbb{R},
$$

at $x$, then at $x$ we have

$$
A^{2}=\frac{\operatorname{tr}(A)}{2} A
$$

(ii) If $\operatorname{rank} A \leqslant 2$ and

$$
A=\alpha g+\epsilon w \otimes w, \quad \alpha \in \mathbb{R}, \quad \epsilon= \pm 1, \quad w \in T_{x}^{*} M,
$$

at $x$ and $w$ is nonzero, then at $x$ we have $\operatorname{rank} A=1$.

Proof. (i) It is clear that (2.5) is equivalent to

$A_{i l}\left(A_{h k} A_{j m}-A_{h m} A_{j k}\right)+A_{j l}\left(A_{i k} A_{h m}-A_{i m} A_{h k}\right)+A_{h l}\left(A_{j k} A_{i m}-A_{i k} A_{j m}\right)=0$.

Contracting this with $g^{h k}$ and $g^{j l}$ we obtain

$$
\operatorname{tr}(A)\left(A_{i l} A_{j m}-A_{i m} A_{j l}\right)+A_{j l} A_{i m}^{2}+A_{i m} A_{j l}^{2}-A_{i l} A_{j m}^{2}-A_{j m} A_{i l}^{2}=0
$$

and (2.6), respectively. Further, substituting (2.7) into (2.10) we get

$$
(\operatorname{tr}(A)-2 \alpha) A \wedge A=2 \beta g \wedge A .
$$

We suppose that $\operatorname{tr}(A)-2 \alpha \neq 0$ at $x$. Now (2.11) yields

$$
A \wedge A=\frac{2 \beta}{\operatorname{tr}(A)-2 \alpha} g \wedge A \text {. }
$$

We note that from (2.5) it follows that $A$ is not proportional to $g$. Thus (2.12), in view of Lemma 3.1 of $[\mathbf{2 1}]$, implies $\beta=0$ and, in a consequence, $\operatorname{rank} A=1$, a contradiction. Therefore $2 \alpha=\operatorname{tr}(A)$. Now (2.11) reduces to $\beta g \wedge A=0$ whence $\beta\left(A-\frac{\operatorname{tr}(A)}{n} g\right)=0$, and in a consequence, $\beta=0$, completing the proof of (i).

(ii) We suppose that (2.5) holds at $x$. From (2.9) we have

$$
\begin{gathered}
A_{i j}=\alpha g_{i j}+\epsilon w_{i} w_{j}, \\
A_{i j}^{2}=\alpha A_{i j}+\epsilon w^{r} A_{r i} w_{j}, \quad w^{r}=g^{r s} w_{s} .
\end{gathered}
$$

(2.14) yields $w^{r} A_{r i} w_{j}=w^{r} A_{r j} w_{i}$ whence

$$
w^{r} A_{r i}=\lambda w_{i}, \quad \lambda \in \mathbb{R} .
$$

Now (2.14) turns into $A_{i j}^{2}=\alpha A_{i j}+\epsilon \lambda w_{i} w_{j}$, which by making use of (2.8) and (2.9) gives $\left(\alpha+\lambda-\frac{\operatorname{tr}(A)}{2}\right) A=\alpha \lambda g$. This implies $\alpha+\lambda=\frac{\operatorname{tr}(A)}{2}$ and $\alpha \lambda=0$. We suppose that $\alpha \neq 0$. Now the last two relations yield

$$
\text { (a) } \quad \lambda=0, \quad(b) \quad \alpha=\operatorname{tr}(A) / 2 .
$$

Evidently, (2.15) by $(2.16)(\mathrm{a})$ reduces to $w^{r} A_{r i}=0$. Now, contracting (2.13) with $g^{i j}$ and transvecting with $w^{j}$, respectively, and using (2.16)(b) we obtain $\frac{n-2}{2} \operatorname{tr}(A)+\epsilon w^{r} w_{r}=0$ and $\operatorname{tr}(A)+\epsilon w^{r} w_{r}=0$, respectively. These relations imply 
$\operatorname{tr}(A)=0$, which by $(2.16)$ (b) yields $\alpha=0$, a contradiction. Since $\alpha=0,(2.9)$ reduces to $A=\epsilon w \otimes w$, completing the proof.

Corollary 2.1. Let $(M, g), n \geqslant 4$, be a semi-Riemannian manifold.

(i) If (1.6) and (1.7) are satisfied on $U_{S} \subset M$, then (1.8) holds on this set.

(ii) If (1.1) and (2.2) are satisfied at every point of $U_{S} \subset M$, then $\operatorname{rank} S=1$ on this set.

Let $M, n \geqslant 3$, be a connected hypersurface isometrically immersed in a semiRiemannian manifold $\left(N, g^{N}\right)$. We denote by $g$ the metric tensor induced on $M$ from the metric tensor $g^{N}$. Further, we denote by $\nabla$ and $\nabla^{N}$ the Levi-Civita connections corresponding to the metric tensors $g$ and $g^{N}$, respectively. Let $\xi$ be a local unit normal vector field on $M$ in $N$ and let $\varepsilon=g^{N}(\xi, \xi)= \pm 1$. We can present the Gauss formula and the Weingarten formula of $(M, g)$ in $\left(N, g^{N}\right)$ in the form: $\nabla_{X}^{N} Y=\nabla_{X} Y+\varepsilon H(X, Y) \xi$ and $\nabla_{X} \xi=-\mathcal{A} X$, respectively, where $X, Y$ are vector fields tangent to $M, H$ is the second fundamental tensor of $(M, g)$ in $\left(N, g^{N}\right), \mathcal{A}$ is the shape operator and $H^{k}(X, Y)=g\left(\mathcal{A}^{k} X, Y\right), k \geqslant 1, H^{1}=H$ and $\mathcal{A}^{1}=\mathcal{A}$. We denote by $R$ and $R^{N}$ the Riemann-Christoffel curvature tensors of $(M, g)$ and $\left(N, g^{N}\right)$, respectively. The Gauss equation of $(M, g)$ in $\left(N, g^{N}\right)$ has the form $R\left(X_{1}, \ldots, X_{4}\right)=R^{N}\left(X_{1}, \ldots, X_{4}\right)+\varepsilon \bar{H}\left(X_{1}, \ldots, X_{4}\right)$, where $\bar{H}=\frac{1}{2} H \wedge H$ and $X_{1}, \ldots, X_{4}$ are vector fields tangent to $M$. Let the equations $x^{r}=x^{r}\left(y^{k}\right)$ be the local parametric expression of $(M, g)$ in $\left(N, g^{N}\right)$, where $y^{k}$ and $x^{r}$ are the local coordinates of $M$ and $N$, respectively, and $a, b, h, i, j, k, l, m \in\{1,2, \ldots, n\}$ and $p, r, t, u \in\{1,2, \ldots, n+1\}$.

Let $M$ be a hypersurface in $N_{s}^{n+1}(c), n \geqslant 4, c=\frac{\tau}{n(n+1)}$, where $\tau$ denote the scalar curvature of the ambient space. Now the Gauss reads (see e.g. [14])

$$
R_{h i j k}=\varepsilon \bar{H}_{h i j k}+\frac{\tau}{n(n+1)} G_{h i j k},
$$

where $R_{h i j k}, G_{h i j k}, H_{h k}$ and $\bar{H}_{h i j k}=H_{h k} H_{i j}-H_{h j} H_{i k}$ denote the local components of the tensors $R, G, H$ and $\bar{H}$, respectively. Contracting (2.17) with $g^{i j}$ we obtain

$$
S_{h k}=\varepsilon\left(\operatorname{tr}(H) H_{h k}-H_{h k}^{2}\right)+\frac{(n-1) \tau}{n(n+1)} g_{h k},
$$

where $\operatorname{tr}(H)=g^{h k} H_{h k}$ and $S_{h k}$ are the local components of the Ricci tensor $S$ of $M$. From (2.18) we easily get

$$
\begin{aligned}
S_{h k}^{2}=g^{i j} S_{h i} S_{k j}= & H_{h k}^{4}-2 \operatorname{tr}(H) H_{h k}^{3}+\left((\operatorname{tr}(H))^{2}-\frac{2(n-1) \varepsilon \tau}{n(n+1)}\right) H_{h k}^{2} \\
& +\frac{2 \varepsilon(n-1) \tau \operatorname{tr}(H)}{n(n+1)} H+\left(\frac{(n-1) \tau}{n(n+1)}\right)^{2} g_{h k} .
\end{aligned}
$$

Further, on every hypersurface $M$ in $N_{s}^{n+1}(c), n \geqslant 4$, we have [19]

$$
R \cdot R-Q(S, R)=-\frac{(n-2) \tau}{n(n+1)} Q(g, C) .
$$


Thus (1.4) is satisfied on every hypersurface in $N_{s}^{n+1}(c), n \geqslant 4$. Evidently, if $x \in U_{R}-U_{H}$, then at $x$ we have $H^{2}=\alpha H+\beta g, \alpha, \beta \in \mathbb{R}$. The last relation leads to (cf. [17, Proposition 3.1(ii)])

$$
R \cdot R=\left(\frac{\tau}{n(n+1)}-\varepsilon \beta\right) Q(g, R) .
$$

Thus (2.3) holds on $U_{R}-U_{H}$. Further, if $M$ is a pseudosymmetric hypersurface in $N_{s}^{n+1}(c), n \geqslant 3$, then on $U_{H} \subset M$ we have [8, Theorem 3.1]

$$
R \cdot R=\frac{\tau}{n(n+1)} Q(g, R) .
$$

It is also known [7, eq. (3.8)] that if $M$ is a pseudosymmetric hypersurface in $N_{s}^{n+1}(c), n \geqslant 3$, then on $U_{S} \subset M$ we have

$$
Q\left(S-\left(L_{R}+\frac{(n-2) \tau}{n(n+1)}\right) g, R-\frac{\tau}{n(n+1)} G\right)=0 .
$$

In particular, applying (2.22) into (2.23) we get on $U_{H} \subset U_{S}$

$$
Q\left(S-\frac{(n-1) \tau}{n(n+1)} g, R-\frac{\tau}{n(n+1)} G\right)=0 .
$$

From this, in view of Lemma 3.4 of [15] it follows that

$$
R-\frac{\tau}{n(n+1)} G=\frac{\phi}{2}\left(S-\frac{(n-1) \tau}{n(n+1)} g\right) \wedge\left(S-\frac{(n-1) \tau}{n(n+1)} g\right),
$$

on the set $V$ of all points of $U_{H}$ at which $S$ has no a decomposition of the form (2.2) and $\phi$ is some function on $V$.

\section{Examples}

Let $(M, g), n \geqslant 4$, be a semi-Riemannian manifold, with nonempty set $U_{C} \cap$ $U_{S} \subset M$, and let its curvature tensor $R$ satisfies on $U_{C} \cap U_{S}$

$$
R=\phi \bar{S}+\mu g \wedge S+\eta G,
$$

where $\phi, \mu$ and $\eta$ are some functions on $U_{C} \cap U_{S}$. According to [9], (3.1) is called the Roter type equation. We mention that above decomposition of $R$ on $U_{C} \cap U_{S}$ is unique [16, Lemma 3.2]. From (3.1) we have [15, Theorem 4.2]: (2.3), with $L_{R}=(n-2)\left(\frac{\mu}{\phi}\left(\mu-\frac{1}{n-2}\right)-\eta\right)$,

$$
\begin{gathered}
R \cdot R-Q(S, R)=\left(L_{R}+\frac{\mu}{\phi}\right) Q(g, C), \\
S^{2}=\left(\kappa+\frac{(n-2) \mu-1}{\phi}\right) S+\frac{\mu \kappa+(n-1) \eta}{\phi} g .
\end{gathered}
$$

Further, as it was shown in [15], (3.1) implies

$$
\begin{aligned}
S_{m}^{r} R_{r i j k}= & (\alpha+\mu)\left(S_{m k} S_{i j}-S_{m j} S_{i k}\right)+\left(\frac{\alpha \mu}{\phi}+\eta\right)\left(g_{i j} S_{m k}-g_{i k} S_{m j}\right) \\
& +\beta\left(g_{m k} S_{i j}-g_{m j} S_{i k}\right)+\frac{\beta \mu}{\phi} G_{m i j k},
\end{aligned}
$$


where $\alpha=\phi \kappa-1+(n-2) \mu, \beta=\mu \kappa+(n-1) \eta$. Now (3.2) leads to (1.5), where

$$
L_{1}=-4(\alpha+\mu), \quad L_{2}=-2\left(\frac{\alpha \mu}{\phi}+\eta+\beta\right), \quad L_{3}=-\frac{4 \beta \mu}{\phi} .
$$

Thus we have

THEOREM 3.1. Every semi-Riemannian manifold $(M, g), n \geqslant 4$, satisfying the Roter type equation is an $A G$ type manifold.

REMARK 3.1. (i) Semi-Riemannian manifolds satisfying $R=\phi \bar{S}$, i.e. the special case of (3.1), were investigated in [24] (see also references therein).

(ii) Examples of warped products satisfying (3.1) are given in [18]. In Example 5.1 of that paper a warped product fulfilling (3.1) is given. That warped product can be locally realized on a hypersurface in a semi-Riemannian space of constant curvature.

(iii) Applying Lemma 3.4 of [15] to (2.23) we conclude that the curvature tensor $R$ of a pseudosymmetric hypersurface $M$ in $N_{s}^{n+1}(c), n \geqslant 4$, is of the form (3.1) at all points of $U_{S} \cap U_{C} \subset M$ at which its Ricci tensor is not of the form (2.2).

(iv) Let $M_{1} \times_{F} M_{2}, p=n-1=\operatorname{dim} M_{1} \geqslant 3, \operatorname{dim} M_{2}=1$, be the warped product defined in [13, Example 4.1]. This manifold satisfies (1.2) and $\operatorname{rank} S=1$. Furthermore, applying the two last relations to (1.3) we get $S \cdot R=0$. The manifold $M_{1} \times_{F} M_{2}$, satisfies $R \cdot R=Q(S, R)$, i.e. (1.4) with $L_{C}=0$. Thus we see that the warped product $M_{1} \times_{F} M_{2}$ is an $A G$ type manifold. This manifold is locally isometric to a hypersurface in a semi-Euclidean space [13, Example 5.1]. We mention that warped products satisfying (1.4) were investigated in [5]. For instance, in [5] it was shown that any warped product $M_{1} \times_{F} M_{2}, \operatorname{dim} M_{1}=1$, $\operatorname{dim} M_{2}=3$, satisfies (1.4).

(v) Let $M_{1} \times{ }_{F} M_{2}, p=\operatorname{dim} M_{1} \geqslant 3, n-p=\operatorname{dim} M_{2} \geqslant 1$, be the warped product defined in Section 4 of [4]. This manifold satisfies $R \cdot R=Q(S, R)$, i.e. (1.4) with $L_{C}=0$, and $\operatorname{rank} S \geqslant n-p+1$. Further, if we assume that $n-p=1$ and the constant $\xi^{f} \xi_{f}$, defined in Section 4 of [4], is nonzero, then $\operatorname{rank} S=2$. Moreover, from (44) of [4] it follows that in this case the scalar curvature $\kappa$ of $M_{1} \times_{F} M_{2}$ is a nonzero constant and (1.7) and (1.8) are satisfied. On such manifolds we also have (1.9) [26, Example 3.1]. Thus, in view of Theorem 3.1, $M_{1} \times_{F} M_{2}$ is an $A G$ type manifold. In addition, this warped product is locally isometric to a hypersurface in a semi-Euclidean space ( [4]; see also [26, Example 4.2]).

(vi) Let $(\bar{M}, \bar{g})$ be a non-flat 2-dimensional Riemannian manifold. It is easy to check that the product manifold $\bar{M} \times \mathbb{E}^{n-2}, n \geqslant 4$, satisfies (1.7), (1.8) and (1.9). Moreover, the manifold $\bar{M} \times \mathbb{E}^{n-2}, n \geqslant 4$, can be realized as a hypersurface in $\mathbb{E}^{n+1}$.

Let $(M, g), n \geqslant 4$, be a semi-Riemannian manifold. We define on $U_{C} \cap U_{S} \subset M$ the tensor $W(R)$ by

$$
W(R)=R-\phi \bar{S}-\mu g \wedge S-\eta G,
$$

where $\phi, \mu$ and $\eta$ are some functions on $U_{C} \cap U_{S}$. The tensor $W(R)$ will be called the Roter type tensor. Manifolds satisfying pseudosymmetry type curvature conditions related to the Roter type tensor will be investigated in subsequent papers. 
We present now an extension of the above definition. Namely, for a generalized curvature tensors $B$ and symmetric $(0,2)$-tensors $A$ and $D$ we define on $U_{\operatorname{Ric}(B)} \cap$ $U_{\text {Weyl }(B)} \subset M$ the $(0,4)$-tensor $W(B, A, D)$ by

$$
W(B, A, D)=B-\phi \bar{A}-\mu A \wedge D-\eta \bar{D},
$$

where $\phi, \mu$ and $\eta$ are some functions on $U_{\operatorname{Ric}(B)} \cap U_{\text {Weyl }(B)}$. The tensor $W(B, A, D)$ will be also called a Roter type tensor. For instance, we have the following Roter type tensors

$$
\begin{gathered}
W(B, A, g)=B-\phi \bar{A}-\mu g \wedge A-\eta G, \\
W(B)=W(B, \operatorname{Ric}(B), g)=B-\phi \overline{\operatorname{Ric}(B)}-\mu g \wedge \operatorname{Ric}(B)-\eta G .
\end{gathered}
$$

Some results on Roter type tensors $W(B, A, g)$ and $W(B, \operatorname{Ric}(B), g)$ are given in $[\mathbf{1 2}]$ and $[\mathbf{2 5}]$. For instance, we have

Proposition 3.1. [25] Let $(M, g), n \geqslant 4$, be a semi-Riemannian manifold admitting a generalized curvature tensor $B$ satisfying $W(B, A, g)=0$ on $U_{\operatorname{Ric}(B)} \cap$ $U_{\mathrm{Weyl}(B)} \subset M$. Then on this set we have

$$
B \cdot B-Q(\operatorname{Ric}(B), B)=L Q(g, \operatorname{Weyl}(B)), \quad L=(n-2)\left(\frac{\mu^{2}}{\phi}-\eta\right) .
$$

Moreover, if $A=\operatorname{Ric}(B)$ on $U_{\operatorname{Ric}(B)} \cap U_{\mathrm{Weyl}(B)}$, then on this set we have

$$
B \cdot B=L_{B} Q(g, B), \quad L_{B}=(n-2)\left(\frac{\mu^{2}}{\phi}-\eta\right)-\frac{\mu}{\phi} .
$$

Proposition 3.2. [12] Let $(M, g), n \geqslant 4$, be a semi-Riemannian manifold admitting a generalized curvature tensor $B$ and let the conditions $B \cdot B=Q(\operatorname{Ric}(B), B)$ $+L Q(g, \operatorname{Weyl}(B))$ and $B \cdot B=L_{B} Q(g, B)$ be satisfied on $U_{\operatorname{Ric}(B)} \cap U_{\mathrm{Weyl}(B)} \subset M$. Then on this set we have

$$
Q\left(\operatorname{Ric}(B)-\left(L_{B}-L\right) g, B-\frac{L}{n-2} G\right)=0 .
$$

Proposition 3.3. [2, Corollary 6.1] Let $(M, g), n \geqslant 4$, be a semi-Riemannian manifold admitting a generalized curvature tensor $B$ and let

$$
Q\left(\operatorname{Ric}(B)-L_{2} g, B-L_{1} G\right)=0
$$

be satisfied on $U=U_{\operatorname{Ric}(B)} \cap U_{\mathrm{Weyl}(B)} \subset M$. Then $W(B)=\phi \overline{\operatorname{Ric}(B)}+\mu g \wedge$ $\operatorname{Ric}(B)+\eta G$ on the subset $V \subset U$ of all points at which the tensor $\operatorname{Ric}(B)$ has no a decomposition in a metrical term and in a term of rank one, where $\phi, \mu$ and $\eta$ are some functions on $V$.

\section{4. $A G$ type hypersurfaces satisfying $\operatorname{rank} S=2$}

Let now $M$ be a hypersurface in $N_{s}^{n+1}(c), n \geqslant 4$, We set [14, eq. (13)]

$$
A=H^{3}-\operatorname{tr}(H) H^{2}+\frac{\varepsilon \kappa}{n-1} H .
$$


Further, let $B$ be a $(0,2)$-tensor with the local components $B_{h k}$ defined by $B_{h k}=$ $g^{i j} H_{h i} S_{k j}$. Using (2.17), (2.18) and (4.1) we obtain

$$
\begin{gathered}
B=-\varepsilon A+\left(\frac{(n-1) \tau}{n(n+1)}+\frac{\kappa}{n-1}\right) H, \\
S \cdot R=-2 \varepsilon H \wedge B-\frac{2 \tau}{n(n+1)} g \wedge S,
\end{gathered}
$$

respectively. Substituting (4.2) into (4.3) and using (2.17) we get

$$
S \cdot R=2 H \wedge A-4\left(\frac{(n-1) \tau}{n(n+1)}+\frac{\kappa}{n-1}\right)\left(R-\frac{\tau}{n(n+1)} G\right)-\frac{2 \tau}{n(n+1)} g \wedge S
$$

Let now $M$ be a Ricci-pseudosymmetric hypersurface in $N_{s}^{n+1}(c), n \geqslant 4$. On $U_{H} \subset M$ we have [3, Theorem 3.1 and Proposition 3.2]

$$
R \cdot S=\frac{\tau}{n(n+1)} Q(g, S) .
$$

It is known (see Proposition 3.2 and Theorem 3.1 of [3]) that (4.5) is equivalent on $U_{H}$ to

$$
H^{3}=\operatorname{tr}(H) H^{2}+\lambda H,
$$

where $\lambda$ is some function on $U_{H}$. Now (4.1) turns into

$$
A=\left(\lambda+\frac{\varepsilon \kappa}{n-1}\right) H .
$$

Applying (2.17) and (4.7) in (4.4) we obtain (cf. [11, Theorem 3.1])

$$
S \cdot R=4\left(\varepsilon \lambda-\frac{(n-1) \tau}{n(n+1)}\right)\left(R-\frac{\tau}{n(n+1)} G\right)-\frac{2 \tau}{n(n+1)} g \wedge S .
$$

If the ambient space is $\mathbb{E}_{s}^{n+1}$, then (2.20) reduces to

$$
R \cdot R=Q(S, R) .
$$

Similarly, in this case, (2.17) reduces to

$$
R_{h i j k}=\varepsilon \bar{H}_{h i j k} .
$$

Proposition 4.1. Let $M$ be a hypersurface in $\mathbb{E}_{s}^{n+1}, n \geqslant 4$. If at $x \in U_{C} \cap$ $U_{S}-U_{H} \subset M$ we have $R \cdot S=0$, then $R \cdot R=0$ at $x$.

Proof. Evidently, (2.21) reduces to $R \cdot R=-\varepsilon \beta Q(g, R)$, which implies $R \cdot S=$ $-\varepsilon \beta Q(g, S)$, and in a consequence, $\beta=0$ at $x$. This completes the proof.

It is clear that every semisymmetric manifold is Ricci-semisymmetric. The converse statement is not true. Under some additional assumptions both conditions are equivalent to each other. This problem, named the problem of P.J. Ryan, was considered by several authors, see $[\mathbf{6}],[\mathbf{1 0}]$ and $[\mathbf{1 1}]$ and references therein. Among other things, in $[\mathbf{6}]$ it was proved that the conditions $R \cdot R=0$ and $R \cdot S=0$ are equivalent on hypersurfaces in $N_{s}^{5}(c)$. 
Proposition 4.2. Let $M$ be a Ricci-semisymmetric an AG type hypersurface in $\mathbb{E}_{s}^{n+1}, n \geqslant 5$, and let the set $U_{H} \subset M$ be nonempty. In addition, let (1.7) be satisfied on $U_{H}$.

(i) The condition $R \cdot R=0$ is satisfied at all points of $U_{H}$ at which $\kappa \neq 0$. Moreover, (1.9) holds at such points.

(ii) The condition $R \cdot R \neq 0$ is satisfied at all points of $U_{H}$ at which $\kappa=0$.

Proof. Let $x \in U_{H}$. From (2.19), in view of Corollary 2.1(i) and (4.6), we get

$$
H^{4}=\left(2(\operatorname{tr}(H))^{2}-\frac{\varepsilon \kappa}{2}\right) H^{2}+\left(2 \lambda+\frac{\varepsilon \kappa}{2}\right) \operatorname{tr}(H) H .
$$

Furthermore, from (4.6) we get

$$
H^{4}=\left((\operatorname{tr}(H))^{2}+\lambda\right) H^{2}+\lambda \operatorname{tr}(H) H .
$$

Comparing the right-hand sides of (4.11) and (4.12) we obtain

$$
\left(\lambda+\frac{\varepsilon \kappa}{2}-\operatorname{tr}(H)\right) H^{2}+\left(\lambda+\frac{\varepsilon \kappa}{2}\right) \operatorname{tr}(H) H=0,
$$

whence $\lambda+\frac{\varepsilon \kappa}{2}=\operatorname{tr}(H)$ and $\left(\lambda+\frac{\varepsilon \kappa}{2}\right) \operatorname{tr}(H)=0$. These relations yield

$$
\text { (a) } \lambda=-\frac{\varepsilon \kappa}{2}, \quad(b) \quad \operatorname{tr}(H)=0 .
$$

Now (4.6) and (4.8) turn into

$$
\begin{gathered}
A=-\frac{\varepsilon(n-3) \kappa}{2(n-1)} H, \\
S \cdot R=-\frac{\kappa}{2} R .
\end{gathered}
$$

respectively. Since $M$ is an $A G$ type manifold, (1.5) holds on $U_{H}$. Now (4.15), by (1.5), leads to

$$
\begin{aligned}
-\frac{\kappa}{2} R_{h i j k}= & L_{1}\left(S_{h k} S_{i j}-S_{h j} S_{i k}\right)+L_{3}\left(g_{h k} g_{i j}-g_{h j} g_{i k}\right) \\
& +L_{2}\left(g_{i j} S_{h k}+g_{h k} S_{i j}-g_{h j} S_{i k}-g_{i k} S_{h j}\right) .
\end{aligned}
$$

If $\kappa \neq 0$ at $x$, then from (4.16), in view of Theorem 4.2 of [15], it follows that (2.3) holds at $x$. Evidently, (2.3) implies (2.4), and in a consequence, we obtain $L_{R}=0$ and $R \cdot R=0$ at $x$. Further, contracting (4.16) with $S_{h}^{l}$ and using (1.8) we obtain

$$
-\frac{\kappa}{2} S_{l}^{h} R_{h i j k}=\left(L_{2}+\frac{\kappa L_{1}}{2}\right) \bar{S}_{l i j k}+\left(L_{3}+\frac{\kappa L_{2}}{2}\right)\left(g_{i j} S_{l k}-g_{i k} S_{l j}\right) .
$$

Symmetrizing this in $l$ and $i$ and using the relation $R \cdot S=0$ we get $\left(L_{3}+\right.$ $\left.\frac{\kappa L_{2}}{2}\right) Q(g, S)=0$, whence

$$
L_{3}=-\frac{\kappa L_{2}}{2} .
$$

On the other hand, contracting (4.16) with $g^{i j}$ and using (1.8) we find

$$
\left(\frac{\kappa}{2}+\frac{\kappa L_{1}}{2}+(n-2) L_{2}\right) S=-\left(\kappa L_{2}+(n-1) L_{3}\right) g,
$$


whence

$$
\text { (a) } \kappa L_{2}=-(n-1) L_{3}, \quad \text { (b) } \frac{\kappa}{2}+\frac{\kappa L_{1}}{2}+(n-1) L_{2}=0 .
$$

From (4.17) and (4.18)(a) we get $L_{3}=0$. Now (4.17) reduces to $L_{2}=0$. Applying this to $(4.18)(\mathrm{b})$ we obtain $\kappa\left(L_{1}+1\right)=0$, whence $L_{1}=-1$. Now (4.16) reduces to (1.9). But this completes the proof of (i).

Let now $\kappa=0$ at $x \in U_{H}$. Thus (4.14) turns into $A=0$. This, together with $(4.13)(\mathrm{b})$, reduces $(2.18)$ and $(4.2)$ to

$$
S_{j k}=-\varepsilon H_{j k}^{2}, \quad B_{h k}=H_{h}^{j} S_{j k}=H_{j k}^{3}=0,
$$

respectively. We suppose that $R \cdot R=0$ at $x$. Now (4.9) yields

$$
\begin{aligned}
& S_{h l} R_{m i j k}+S_{i l} R_{h m j k}+S_{j l} R_{h i m k}+S_{k l} R_{h i j m} \\
& -S_{h m} R_{l i j k}-S_{i m} R_{h l j k}-S_{j m} R_{h i l k}-S_{k m} R_{h i j l}=0 .
\end{aligned}
$$

This, by transvection with $H_{a}^{l}$ and $H_{b}^{h}$ and making use of (4.10) and (4.19), leads

$$
S_{i m}\left(S_{b j} S_{l k}-S_{b k} S_{l j}\right)+S_{i l}\left(S_{b j} S_{k m}-S_{b k} S_{j m}\right)=0 .
$$

We set $Y_{k}=X^{j} S_{j k}$, where $X^{j}$ and $Y^{j}$ are the local components of vectors $X, Y \in$ $T_{x} M$ such that $Y_{1}^{2}+\cdots+Y_{n}^{2}>0$, where $Y_{k}=g_{j k} Y^{j}$. Transvecting now (4.20) with $X^{l}$ and $X^{m}$ we obtain $Y_{i}\left(Y_{k} S_{b j}-Y_{j} S_{b k}\right)=0$, whence it follows that rank $S=1$ at $x$, a contradiction. Thus if $\kappa=0$ at $x \in U_{H}$, then $R \cdot R \neq 0$ at $x$. Our proposition is thus proved.

The last proposition implies

TheOREM 4.1. Let $M$ be an $A G$ type hypersurface in $\mathbb{E}_{s}^{n+1}, n \geqslant 5$, satisfying (1.7) on nonempty $U_{H} \subset M$. The conditions $R \cdot R=0$ and $R \cdot S=0$ are equivalent on the subset of $U_{H}$ of all points at which $\kappa \neq 0$.

\section{References}

[1] M.A. Akivis and V.V. Goldberg, Semiintegrable almost Grassmann structures, Differential Geom. Appl. 10 (1999), 257-294.

[2] M. Belkhelfa, R. Deszcz, M. Głogowska, M. Hotloś, D. Kowalczyk, and L. Verstraelen, A review on pseudosymmetry type manifolds, in: Banach Center Publ. 57, Inst. Math., Polish Acad. Sci., 2002, 179-194.

[3] F. Defever, R. Deszcz, P. Dhooghe, L. Verstraelen, and S.. Yaprak, On Ricci-pseudosymmetric hypersurfaces in spaces of constant curvature, Results in Math. 27 (1995), 227-236.

[4] F. Defever, R. Deszcz, M. Głogowska, V.V. Goldberg, and L. Verstraelen, A class of fourdimensional warped products, Demonstratio Math. 35 (2002), 853-864.

[5] F. Defever, R. Deszcz, and M. Prvanović, On warped product manifolds satisfying some curvature condition of pseudosymmetry type, Bull. Greek Math. Soc. 36 (1994), 43-67.

[6] F. Defever, R. Deszcz, Z.Sentürk, L. Verstraelen, andS. Yaprak, On a problem of P.J. Ryan, Glasgow Math. J. 41 (1999), 271-281.

[7] R. Deszcz, On pseudosymmetric hypersurfaces in spaces of constant curvature, Tensor, (N.S.), 58 (1997), 253-269.

[8] R. Deszcz, On certain classes of hypersurfaces in spaces of constant curvature, in: Geometry and Topology of Submanifolds, III, World Sci. Publishing, River Edge, NJ, 1991, 131-147. 
[9] R. Deszcz, On curvature properties of hypersurfaces in space forms, the talk given at the conference Conference on Geometry held at the Mathematical Research and Conference Center in Bȩdlewo, 16-21 September 2002.

[10] R. Deszcz and M. Głogowska, Examples of nonsemisymmetric Ricci-semisymmetric hypersurfaces, Colloq. Math. 94 (2002), 87-101.

[11] R. Deszcz and M. Głogowska, Some nonsemisymmetric Ricci-semisymmetric warped product hypersurfaces, Publ. Inst. Math. (Beograd) (N.S.), 72 (86) (2002), 81-94.

[12] R. Deszcz, M. Głogowska, M. Hotloś, D. Kowalczyk, and L. Verstraelen, A review on pseudosymmetry type manifolds, Dept. Math., Agricultural Univ. Wrocław, Ser. A, Theory and Methods, Report No. 84, 2000.

[13] R. Deszcz, M. Głogowska, M. Hotloś, and Z.Ṣentürk, On certain quasi-Einstein semisymmetric hypersurfaces, Ann. Univ. Sci. Budapest. Eötvös Sect. Math. 41 (1998), 151-164.

[14] R. Deszcz, M. Głogowska, M. Hotloś, and L. Verstraelen, On some generalized Einstein metric conditions on hypersurfaces in semi-Riemannian space forms, Colloq. Math. 96 (2003), 149166 .

[15] R. Deszcz and M. Hotloś, On a certain subclass of pseudosymmetric manifolds, Publ. Math. Debrecen 53 (1998), 29-48

[16] R. Deszcz and M. Hotloś, On hypersurfaces with type number two in space forms, Dept. Math., Agricultural Univ. Wrocław, Ser. A, Theory and Methods, Report No. 102, 2002.

[17] R. Deszcz, M. Hotloś, and Z.Ṣentürk, Quasi-Einstein hypersurfaces in semi-Riemannian space forms, Colloq. Math. 89 (2001), 81-97.

[18] R. Deszcz and D. Kowalczyk, On some class of pseudosymmetric warped products Colloq. Math. 97 (2003), 7-22.

[19] R. Deszcz and L. Verstraelen, Hypersurfaces of semi-Riemannian conformally flat manifolds, in: Geometry and Topology of Submanifolds, III, World Sci. Publishing, River Edge, NJ, 1991, 131-147.

[20] M. Głogowska, Curvature properties of some four-dimensional manifolds, Demonstratio Math. 34 (2001), 901-918.

[21] M. Głogowska, Semi-Riemannian manifolds whose Weyl tensor is a Kulkarni-Nomizu square, Publ. Inst. Math. (Beograd) (N.S.), 72 (86) (2002), 95-106.

[22] M. Głogowska, Curvature conditions of pseudosymmetric type on hypersurfaces with two distinct principal curvatures, Dept. Math., Agricultural Univ. Wrocław, Ser. A, Theory and Methods, Report No. 108, 2003.

[23] M. Głogowska, On quasi-Einstein Cartan type hypersurfaces, to appear.

[24] D. Kowalczyk, On some subclass of semisymmetric manifolds, Soochow J. Math. 27 (2001), 445-461.

[25] D. Kowalczyk, On the Schwarzschild type spacetime, Dept. Math., Agricultural Univ. Wrocław, Ser. A, Theory and Methods, Report No. 83, 2000.

[26] C. Murathan, K. Arslan, R. Deszcz, R. Ezentaș, and C.Özgür, On a certain class of hypersurfaces of semi-Euclidean spaces, Publ. Math. Debrecen 58 (2001), 587-604.

Department of Mathematics

(Received 1102 2003) Agricultural University of Wrocław Grunwaldzka 53, 50-357 Wrocław

Poland

rysz@ozi.ar.wroc.pl 